\title{
Estudiantes con déficit atencional enfrentando tareas de comprensión lectora y producción de textos: Un estudio comparativo en alumnado de $4^{\circ}$ año de educación primaria en Chile
}

\author{
Students with Attention Deficit Disorder Carrying out Tasks of Reading Comprehension and Text \\ Production: A Comparative Study in 4th-Grade Students of Primary Education in Chile

\begin{abstract}
Crianças com déficit de atenção fazendo tarefas de compreensão de leitura e produção de texto: Um estudo comparativo em estudantes do $4^{\circ}$ ano do ensino fundamental no Chile
\end{abstract}

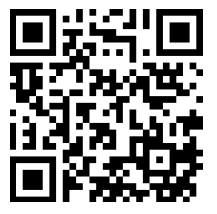

\author{
Fabián Andrés Inostroza-Inostroza \\ Universidad Alberto Hurtado \\ Santiago, Chile \\ fabian.uah.doc@gmail.com \\ Dttp://orcid.org/0000-0003-1530-2266
}

Recibido 26 de julio de 2016 • Corregido 14 de junio de 2017 • Aceptado 25 de agosto de 2017

Received 26 de julio de 2016 • Revised 14 de junio 2017 • Accepted 25 de agosto de 2017

Recebido 26 de julio de 2016 • Revisado 14 de junio 2017 • Aprovado 25 de agosto de 2017

\begin{abstract}
Resumen: El presente artículo tiene como objetivo comparar el desempeño de estudiantes que presentan la condición de trastorno de déficit atencional y quienes no la presentan, en tareas de comprensión lectora y producción de textos en cuarto año de educación primaria. Se busca, por medio de este estudio cuantitativo, no experimental de carácter comparativo, proveer evidencia respecto de la manera en la cual esta condición limita los resultados de aprendizaje en las tareas de comprensión y producción de textos, lenguaje y comunicación. Dentro de los principales hallazgos derivados de esta investigación, se encuentra un menor desempeño en comprensión lectora del estudiantado con déficit atencional respecto a sus pares que no presentan este diagnóstico. Esta misma tendencia se observa en los resultados en tareas de producción de textos. Lo anterior implica que esta condición, que afecta la atención del estudiantado, interfiere en el proceso del aprendizaje del lenguaje en el caso de la niñez que lo presenta, por lo que genera desafíos y nuevas preguntas para quienes investigan esta temática y, para el personal docente plantea nuevos retos a la hora de planificar la enseñanza en el área del lenguaje con el propósito de adaptarla a los requerimientos específicos de este alumnado.
\end{abstract}

Palabras claves: Trastorno de déficit atencional; comprensión lectora; producción de textos; dificultades del aprendizaje.

\footnotetext{
${ }^{1}$ Licenciado en Educación, Profesor de Educación General Básica por la Pontificia Universidad Católica de Chile (2011), Magíster en Educación mención Dificultades del Aprendizaje por la Pontificia Universidad Católica de Chile (2015). Actualmente cursando estudios de Doctorado en Educación por las universidades Alberto Hurtado y Diego Portales (2016). Ejerció durante 5 años como profesor de primaria en establecimientos municipales y particulares de la ciudad de Santiago en Chile. Participó en proyectos de investigación estatales como ayudante y tesista de Magíster (proyectos FONDECYT). Actualmente es revisor de la revista Iberoamérica Social - Red de estudios Sociales en el área de educación. Ha publicado en temáticas como: educación y discapacidad, matemáticas y exclusión, dificultades de aprendizaje en el área de las matemáticas, proyectos de integración escolar y necesidades educativas especiales.
} 
doi: http://dx.doi.org/10.15359/ree.21-3.7

URL: http://www.una.ac.cr/educare

CORREO: educare@una.cr

\begin{abstract}
The present article aims to compare the performance in students with Attention Deficit Disorder to those who do not present it, in tasks of reading comprehension and text production carried out by students attending the fourth grade of primary education. This quantitative, nonexperimental comparative study aims to provide evidence regarding the way in which this condition limits the learning outcomes in the tasks of comprehension and production of texts, language, and communication. One the main findings of this research was that students with attention deficit disorder showed lower reading comprehension performance than their peers who do not presented this diagnosis. This same tendency was observed in the results of the texts production tasks. This implies that the disorder affecting the attention in students with it interferes in the process of language learning. This generates challenges and new questions for those who do research in this topic; and for the case of teachers, it presents new challenges when planning the teaching in order to adapt it to the specific requirements of these students in the area of language.
\end{abstract}

Keywords: Attention deficit disorder, reading comprehension, text production, learning disabilities.

Resumo: O objetivo deste artigo é fazer uma comparação entre o desempenho de estudantes com o transtorno de déficit de atenção e aqueles que não o têm, na realização de tarefas de compreensão de leitura e produção de texto, no quarto ano do ensino fundamental. Através deste estudo quantitativo, não experimental e de caráter comparativo, busca-se fornecer evidências sobre a maneira pela qual este transtorno limita os resultados da aprendizagem, quando realizam tarefas de compreensão e produção de textos, linguagem e comunicação. Entre as principais conclusões desta investigação, existe um baixo desempenho na compreensão de leitura dos alunos com transtorno de déficit de atenção em relação aos alunos que não têm esse diagnóstico. Esta mesma tendência é vista nos resultados de tarefas de produção de texto. Isto implica que esta condição, que afeta a atenção da criança, interfere no processo de aprendizagem da linguagem na infância. Este fator cria desafios e novas questões para pessoas que pesquisam esta temática e para o corpo docente, desafiado a ter que re-elaborar o planejamento de aula na área de linguagem, a fim de adaptá-lo às necessidades específicas desses estudantes.

Palavras-chave: Transtorno de Déficit de Atenção; compreensão de leitura; produção de textos, dificuldades de aprendizagem.

\title{
Introducción
}

El presente informe de investigación indaga desde un enfoque cuantitativo de investigación, respecto del desempeño de estudiantes que presentan la condición de TDAH (trastorno de déficit de atención, de aquí en adelante) en el desempeño en tareas de comprensión lectora ( $C L$ de aquí en adelante) y producción de textos (PT de aquí en adelante). Compara el desempeño en estudiantes de cuarto año básico con TDAH y sin TDAH de una escuela particular subvencionada de la comuna de Macul de la ciudad de Santiago de Chile.

El propósito fundamental de este estudio es aportar información relevante y pertinente al sistema educativo en general, a los grupos investigadores que desarrollan sus líneas de investigación e interés en esta área y para docentes con el desafío de adecuar sus prácticas pedagógicas en función del respeto a la diversidad presente en sus aulas. 
En cuanto al diseño de investigación empleado, este corresponde a un diseño no experimental, llamado ex post facto (después de los hechos), ya que no se tiene control de la variable independiente (TDAH). En cuanto a las decisiones muestrales, el grupo experimental corresponde al estudiantado diagnosticado con TDAH, informado este diagnóstico a través de las educadoras diferenciales del Proyecto de Integración Escolar (PIE de aquí en adelante) y para el caso del grupo control se escogieron, al azar, a 5 estudiantes de cada $4^{\circ}$ año básico del establecimiento (4 cursos en este nivel, 20 estudiantes en total en el grupo control).

La pregunta de investigación que orienta este estudio corresponde a: ¿De qué manera afecta la condición de déficit atencional en el desempeño en tareas de comprensión lectora (CL) y producción de textos (PT) en estudiantes de $4^{\circ}$ año de enseñanza básica con diagnóstico por esta condición?

La relevancia teórica de este estudio radica en la evidencia empírica que se puede extraer para indagar cuantitativamente si la condición de TDAH afecta en el desempeño en tareas de CL y PT, lo tendría repercusiones en otras áreas curriculares como es el caso de las ciencias sociales, las ciencias naturales, las matemáticas, entre otras asignaturas. Pero más allá del ámbito escolar, este trastorno puede tener incidencia en el cotidiano del alumnado y futuros adultos y adultas en su desarrollo personal, académico y laboral.

Una de las hipótesis que ha surgido por parte del personal directivo del establecimiento y que es compartida por el personal docente en el establecimiento en donde se desarrolló esta investigación, es que los desempeños en general descendidos en las áreas de CL y PT, se deben a la alta presencia de estudiantes que pertenecen al PIE dentro de cada curso y específicamente de aquel alumnado diagnosticado por TDAH.

Por tanto, es de sugerente interés establecer la incidencia del TDAH en la desempeño de estudiantes que han recibido tal diagnóstico (grupo experimental), en las tareas de CL y PT, respecto al estudiantado que no presentan este cuadro (grupo control), para poder definir si el estudiantado con TDAH, efectivamente, presenta niveles más descendidos en las áreas de $\mathrm{CL}$ y $\mathrm{PT}$, que sus pares sin esta condición.

De manera preliminar en esta investigación se realizará una comparación entre los rendimientos del estudiantado dentro del nivel educativo en cuestión, es decir, en $4^{\circ}$ año básico $\left(4^{\circ} \mathrm{A}, 4^{\circ} \mathrm{B}, 4^{\circ} \mathrm{C}\right.$ y $\left.4^{\circ} \mathrm{D}\right)$, para analizar si efectivamente el estudiantado con TDAH presenta un rendimiento inferior al de sus pares en tareas de $\mathrm{CL}$ y PT. Lo anterior se pondrá a prueba a través de un instrumento denominado "Pruebas de comprensión lectora y producción de textos (CLPT)"; diseñado y estandarizado para Chile por las autoras Medina y Gajardo (2010). El detalle sobre estos aspectos se presentará en el apartado de "diseño" que incluirá más adelante en este informe. 
doi: http://dx.doi.org/10.15359/ree.21-3.7

URL: http://www.una.ac.cr/educare

CORREO: educare@una.cr

\section{Antecedentes del estudio}

Es fundamental para la educación primaria la comprensión lectora y la producción de textos por parte del estudiantado, ya que, de acuerdo con Vallés (2005), la CL corresponde a:

Herramienta vital para acceder al conocimiento y la construcción de la realidad, ya que es una competencia vital en el procesamiento de la información y generadora de otras competencias tales como el análisis, la imaginación, la deducción, la lectura de códigos, símbolos, entre otras. (p. 49)

Por otro lado, se puede aseverar lo mismo respecto a la PT, ya que sin esta habilidad el estudiantado no podría expresar, por medio de la palabra escrita, sus pensamientos o el resultado del proceso de razonamiento, lo que limitaría su participación en el mundo educativo y laboral, como también sería una barrera que los excluiría de una participación ciudadana efectiva (Medina y Gajardo, 2010).

Sumado a lo anterior, existe evidencia internacional, aportada por el Estudio sobre el comportamiento lector a nivel nacional, elaborado por la Universidad de Chile (Universidad de Chile, Centro Micro Datos, 2011), que señala que a nivel OCDE, Chile se encuentra en los últimos lugares en esta habilidad lingüística. En el ámbito nacional se constata este hecho, a través del desempeño obtenido por el alumnado en la prueba censal SIMCE' (2012) y Pruebas de CL- PT (Medina y Gajardo, 2010), que señalan que el estudiantado chileno obtiene niveles descendidos en ambas tareas, pero principalmente en CL. Es así como las autoras Medina y Gajardo (2010) postulan, en esta misma línea, respecto a los estudios a nivel internacional: Estos revelan que el nivel de comprensión lectora y de producción de textos en escolares chilenos es insuficiente, comparado con los resultados de otros países.

Las cifras más actualizadas en el ámbito de la $\mathrm{CL}$ que ofrece la última medición a nivel nacional en la prueba SIMCE arrojó como resultado que el puntaje promedio nacional en $4^{\circ}$ año básico fue de 264 puntos, disminuyendo en 3 puntos respecto a la medición del año anterior (2012), mientras que en la misma prueba (CL) pero aplicada en el nivel de $I^{\circ}$ año de educación media el puntaje promedio alcanzó los 254 puntos, evidenciando una baja en 5 puntos respecto a la medición anterior (año 2012). Lo anteriormente expuesto indica que el nivel de $\mathrm{CL}$ del estudiantado no tiende a mejorar y se encuentra en niveles de desempeño insuficiente y elemental (Agencia de Calidad, Gobierno de Chile, 2016).

De forma más específica, el porcentaje de estudiantes que se encuentra en los niveles de aprendizaje elemental e insuficiente, corresponde a $62,6 \%$ en el caso de $4^{\circ}$ básico (Agencia deCalidad, Gobierno de Chile, 2016), lo que indica que casi dos tercios de los estudiantes que cursaban este nivel

2 Sistema de Medición de la Calidad de la Educación, prueba de carácter censal aplicada a estudiantes de $4^{\circ}$ año básico y en otros niveles educativos en donde se evalúan los aprendizajes logrados en las asignaturas de matemáticas, lenguaje y ciencias. 
educativo en el momento de la aplicación de la prueba no alcanzan un nivel de comprensión lectora satisfactoria. En los términos en que el Ministerio de Educación lo expone:"[estos alumnos y alumnas] no logran demostrar consistentemente que han adquirido los conocimientos y las habilidades más elementales estipulados en el currículum para el período evaluado" (Agencia de Calidad, Gobierno de Chile, 2013, p. 6). Respecto a estudiantes que se encuentran en el nivel elemental, señala al respecto: "han logrado lo exigido en el currículum de manera [parcial]. Esto implica demostrar que han adquirido los conocimientos y las habilidades [más elementales] estipulados [por] el currículum en el período evaluado" (Agencia de Calidad, Gobierno de Chile, 2013, p. 6).

En cuanto a los conceptos relevantes en este estudio, el TDAH, la prevalencia que tiene esta condición es alta respecto a otras DEA (dificultades especificas del aprendizaje), y se encuentra como uno de los diagnósticos con mayor frecuencia en la población en edad escolar. Existen diferentes estudios que en promedio informan que su prevalencia es del $5 \%$ en el caso de EE. UU (Faraone, Sergeant, Gillberg, y Biederman, 2003). En el país, los estudios señalan que la presencia de este cuadro oscila entre el 6 y el 9\% (López, 2006). Existe acuerdo en las investigaciones en lo relativo a que este síndrome tenderá a aumentar su prevalencia a medida que pase el tiempo y que precisamente este cuadro tiende a afectar el rendimiento académico del estudiantado (Carvajal, Cabezas y Valenzuela, 2006), por tanto, se hace preciso profundizar en su relación con el desempeño del estudiantado con esta etiqueta, específicamente en las áreas de CL y PT.

La evidencia encontrada en la bibliografía que realiza el vínculo el TDAH con las competencias comunicativas y en específico con la $\mathrm{CL}$, apunta que el $50 \%$ de niñez con este cuadro tiene problemas en el ámbito de la comprensión del lenguaje escrito, lo que incide negativamente en el rendimiento global y en el nivel de educación que alcanzan (Miranda-Casas, Fernández-Andrés, Robledo y García-Castellar, 2010). En cuanto a la producción de textos, existen estudios a nivel nacional que señalan que tienen dificultades en tareas que impliquen organizar el discurso oral y escrito a nivel sintáctico respecto a sus pares sin este cuadro (Elías, Crespo y Góngora, 2012).

Dados estos antecedentes, es indispensable indagar de forma más específica en la incidencia del TDAH en las tareas de CL y PT, aspectos que serán discutidos con mayor amplitud en el marco teórico, en donde se establecerán las definiciones que se adoptarán en el marco de esta investigación y la forma en que se operacionalizarán las variables para ser susceptibles de ser medidas.

\section{Marco teórico}

Este apartado se centrará en ofrecer definiciones conceptuales y se optará por escoger aquellas que sean más coherentes con los objetivos de la investigación como también pertinentes en cuanto a este estudio.

La búsqueda bibliográfica se realizó considerando las investigaciones presentes en bases de datos como Web of Science, Scielo, EBSCO y ERIC, en revistas de corriente principal indexadas en estas bases, en textos, manuales, etc. Los criterios de inclusión fueron los siguientes: 
doi: http://dx.doi.org/10.15359/ree.21-3.7

URL: http://www.una.ac.cr/educare

CORREO: educare@una.cr

a) Criterio temporal: bibliografía disponible dentro de los últimos 20 años sobre los temas asociados a esta investigación.

b) Criterio de pertinencia: los textos, revistas, manuales, libros, etc. tenían que tratar como temáticas centrales, las asociadas con la comprensión lectora, la producción de textos y el trastorno por déficit de atención con hiperactividad. En caso de que trataran estos conceptos de manera tangencial o secundaria, no se consideraba dicha bibliografía.

c) Criterio de contextualización: los textos analizados debían estar asociados al área de educación o psicopedagogía y en lo posible que muestran resultados de investigación en el contexto latinoamericano.

Con base en esta revisión, preliminarmente se seleccionaron 36 textos, de los cuales se recogieron los aportes de 20 de ellos.

\section{Rendimiento académico}

El rendimiento académico se conceptualiza como un constructo que implica una serie de articulaciones cognitivas generadas por los sujetos que sintetiza atributos de cantidad y cualidad como factores de medición y predicción de la experiencia educativa. Lo anterior considerando la constelación dinámica de atributos, los que pueden ser la motivación escolar, el autocontrol y las habilidades sociales que acopladas pueden dar cuenta de los resultados de un proceso de aprendizaje (Edel, 2003).

De la misma forma, otros estudios argumentan que el rendimiento académico no es solo el producto de la actividad cognitiva de los sujetos, sino que adicionalmente se deben considerar los factores afectivos y emocionales, como también resaltar la importancia que tienen los factores relacionados con el aula (relación profesorado -alumnado, clima de aula) y los contextuales (entorno familiar, capital cultural familiar, relaciones sociales de estudiantes). Por tanto, el rendimiento académico, si bien da cuenta de un proceso de aprendizaje, este va más allá del reduccionismo implícito de algunas investigaciones que asocian a un resultado cuantitativo el producto de todo un proceso educativo (García, Alvarado y Jiménez, 2000).

En este sentido, sin duda alguna este constructo ha sido parte de debates epistemológicos y conceptuales, debido a que no es unívoca su definición. En la mayoría de los casos se reconoce que va más allá de un indicador, como una calificación, por ejemplo, pero a que a pesar de este consenso, generalmente se tiende a estimar el rendimiento en términos académicos de forma cuantitativa por medio de un reductor de complejidad, que se traduce en una expresión numérica.

Para el caso de esta investigación, se considera que el rendimiento académico en tareas de comprensión lectora y producción de textos va a ser medido por medio de indicadores 
cuantitativos, que en consonancia con lo propuesto con las investigaciones mencionadas refleja una constelación de variables más allá de la mera actividad cognitiva asociada al lenguaje. En este sentido, aplicar un instrumento validado y adaptado al sistema educativo chileno permite reducir la complejidad que implicaría aplicar otro tipo de instrumentos en que se consideran todas las dimensiones del proceso de aprendizaje de la lecto-escritura. Esta decisión está fundada principalmente en términos de la factibilidad de llevar a cabo este diseño ex post facto.

\section{Comprensión lectora (CL)}

Ante una multiplicidad de definiciones, se llega a un cierto consenso que establece que la $C L$ es un proceso complejo en el cual están involucradas diversas habilidades que permiten al sujeto lector extraer el significado de un texto escrito. Una de línea de estudios que se ha encargado de estudiar la CL es la corriente cognitiva de la psicología, desde la cual se entenderá la CL como un proceso en el cual están involucradas múltiples tareas mentales. Como una forma de reafirmar la aseveración anterior, los investigadores Cain, Oakhill y Bryant (2004) proponen que la comprensión de textos es una tarea compleja que se basa en diferentes habilidades y procesos cognitivos.

Las autoras Medina y Gajardo (2010), recogiendo y sintetizando los aportes de múltiples visiones, concluyen:

Durante la lectura, se produce una transacción, entre el lector y el texto (Rossenblatt, 1978), en el cual el que lee, a partir de las claves del contexto y del texto, activa sus conocimientos y experiencias para a portarlos al proceso lector, establece relaciones, anticipa el contenido de lo escrito, formula hipótesis, realiza inferencias, se plantea interrogantes, elabora respuestas que responden a su propósito de lectura [y construye nuevos conocimientos en la interacción entre el texto y los conocimientos previos del lector] (p. 63).

La conceptualización de CL que se empleará en esta investigación será la de Medina y Gajardo (2010), la que integra los aportes de la mirada cognitiva y constructivista de este proceso. Adicionalmente permitirá realizar una mejor operacionalización de este constructo teórico.

Los rasgos o dimensiones que evaluará el instrumento escogido, en cuanto a la $\mathrm{CL}$, son: “1) Comprensión de estructuras y funciones textuales, 2) comprensión literal, 3) comprensión inferencial, 4) Comprensión crítica, 5) Comprensión metacognitiva, 6) Reorganización de la información y 7) manejo del código" (Medina y Gajardo, 2010, p. 108). 
doi: http://dx.doi.org/10.15359/ree.21-3.7

URL: http://www.una.ac.cr/educare

CORREO: educare@una.cr

\section{Producción de textos (PT)}

Dentro de la bibliografía existente y revisada en cuanto a PT, en la mayoría de las investigaciones existe un acuerdo respecto a que este proceso corresponde a un fenómeno de naturaleza compleja y que es una habilidad que requiere de una instrucción intencionada de forma tal que una vez que sea efectivamente aprendida, sea empleada para servir a múltiples propósitos sociales. Es más, puede ser pensada como una tecnología (Rubio, 2011 citando a Pozo, 2001, p. 125), es decir:

[Como] un artefacto cultural cuyo dominio requiere de esfuerzo consciente y práctica constante (Grabe \& Kaplan, 1996; Casanny, 1999), que cumple, en gran medida la función de constituirse como un sistema de memoria externa que, a su vez, impacta en el desarrollo de la cognición humana".

Respecto a la PT se evidenciaron aportes provenientes de la psicología cognitiva que explican el cómo una persona, en este caso, estudiante, construirá un texto con base en cuatro grandes etapas, las que en orden de desarrollo corresponden a: "Planificación del mensaje, construcción ... sintáctica, selección de palabras, y procesos motores" (Cuetos, 2009, p. 24).

Desde un enfoque sociocultural de la PT, esta es entendida, según Cassany (2006, citado por Medina y Garjardo, 2010), en los siguientes términos:

Escribir es una actividad humana que apunta a conseguir un objetivo dentro de una comunidad discursiva dada, que está altamente vinculada con el yo, con la mente, con la imagen social, con la comunidad a la cual se pertenece, con la disciplina o con el grupo de pertenencia. (p. 67)

Otra perspectiva situada en el contexto educacional chileno es la que van a adoptar las autoras Medina y Gajardo (2010), quienes recogiendo múltiples visiones respecto a la producción de textos y realizando la intersección con la noción que se empleó en la prueba experimental SIMCE en el año 2008 de Producción de Textos, logran proponer una conceptualización de este proceso, el que definen de la siguiente forma:

Es un proceso cognitivo complejo y múltiple que pretende solucionar un problema comunicativo en situaciones reales, a través de la creación de un texto. Las habilidades involucradas son la adecuación a la situación comunicativa, la organización textual, la coherencia y cohesión del texto, y el uso de las convenciones de la lengua escrita. (p. 262)

Respecto a la elección de una definición conceptual que sea la más completa e integre aspectos relevantes para esta investigación, se adoptó la propuesta de Medina y Gajardo (2010), debido a que incorpora más dimensiones que el modelo propuesto por Cuetos (2009), además 
es mucho más específica que la definición que proporciona Rubio (2011) y, por último, está alineada con las exigencias del actual marco curricular chileno y además incorpora los contenidos relevantes que consideró la prueba experimental SIMCE 2008 a los $4^{\circ}$ básicos para evaluar la PT.

Por los motivos señalados con anterioridad es que se ha considerado que es mucho más pertinente a la realidad de las escuelas chilenas, ya que se han hecho pilotajes de este instrumento en los años 2009 y 2010, en primer y segundo ciclo de enseñanza básica en escuelas de distintas dependencias y regiones del país, con resultados positivos en cuanto a validez y confiablidad del constructo y el instrumento.

Los rasgos para operacionalizar la PT, de acuerdo con Medina y Gajardo (2010), son los siguientes: 1) Adaptación a la situación comunicativa, 2) Ideas, 3) Voz o sello personal, 4) Elección de palabras, 5) Fluidez y cohesión, 6) Estructura y organización y 7) Convenciones gramaticales.

\section{El trastorno de atención con hiperactividad o sin esta o síndrome de déficit de atención}

Más allá de la multiplicidad de definiciones de este síndrome, la que se presenta de forma predominante en la bibliografía revisada es aquella que considera esta condición desde un paradigma clínico (o biomédico), es decir, se presume que es un cuadro cuyo sustrato (o etiología) es biológico, por tanto, el diagnóstico diferencial debiese estar alineado con dicha concepción (López, 2006).

Con el fin de reafirmar lo anteriormente planteado, el investigador López (2006) señala respecto al proceso de identificación del cuadro: "El diagnóstico del TDAH ha sido y continúa siendo clínica ... en él, se reconocen características fenotípicas conductuales cuyos síntomas nucleares son derivados de las dificultades de atención, control de impulsos e hiperactividad" (p. 14).

Otra perspectiva, situada en el mismo enfoque clínico, es la que ofrecen las investigaciones de Francisco Aboitiz, autor que genera una línea de investigación filogenética, es decir, realiza un estudio longitudinal para verificar que el TDAH es una respuesta a las condiciones ambientales cambiantes del ser humano en los últimos tres mil años. De hecho, su hipótesis de trabajo es "que el TDAH corresponde a una estrategia atencional que fue seleccionada en el pasado y que ha sido gradualmente remplazada por mecanismos que refuerzan la capacidad de concentración y de diferir temporalmente la expectativa de recompensa" (Aboitiz, López-Calderón, López y Galaburda, 2006, p. 27).

Tanto las definiciones ofrecidas por López (2006) como el aporte de Aboitiz et al. (2006) apuntan a que el cuadro sería entendido desde una perspectiva clínica y a la vez comparten que las tres grandes dimensiones en las que se puede desglosar este cuadro serían: inatención, hiperactividad e impulsividad. 
doi: http://dx.doi.org/10.15359/ree.21-3.7

URL: http://www.una.ac.cr/educare

CORREO: educare@una.cr

Otra mirada en torno al TDAH es la que ofrece Condemarín, Gorostegui y Milicic (2008), desde la cual los aspectos psicológicos y psicopedagógicos son relevados, por tanto, este grupo investigativo define este cuadro como "un patrón persistente de hiperactividad/impulsividad más frecuente y severo de lo habitualmente observado en individuos de un nivel de desarrollo comparable" (p.16).

La definición anterior agrupa a niños con inteligencia "normal" y destaca como característica distintiva: "una desviación significativa de la norma en tres síntomas cardinales: inatención, impulsividad e hiperactividad, que llevan a dificultades permanentes y de inicio temprano en su adaptación social y/o [sic] rendimiento, en relación a [sic] su edad de desarrollo" (Condemarín et al., 2008, p. 17).

Para los fines de este estudio se ha seleccionado una definición que es consistente con las investigaciones tanto a nivel nacional como internacional, una conceptualización que resalta que los síntomas cardinales de este síndrome corresponden a una combinación de inatención, impulsividad e hiperactividad que están presentes tempranamente en la vida del niño, pero que se hacen más evidentes en la edad escolar. Esta definición propone la visión psicopedagógica ofrecida por Condemarín et al. (2008), sin sustraerse al hecho de que los aportes de Aboitiz et al. (2006) desde neurobiología y de López (2006) desde la psiquiatría también son valiosos, pero no son conocimientos científicos construidos para ser aplicados al ámbito de la educación.

\section{EI TDAH y su incidencia en el rendimiento académico y en el área de lenguaje}

De acuerdo con la bibliografía revisada, existe consenso en cuanto a que el TDAH incide de forma negativa en el proceso de enseñanza y aprendizaje y en el rendimiento de estudiantes que presentan este cuadro durante su periodo de escolaridad (Carvajal et al., 2006).

En términos globales se puede establecer que un niño con TDAH:

Puede presentar interferencias en el aprendizaje por la atención dispersa, por la dificultad de integrar contenidos, por la dificultad de retener información y por la falta de reflexión previa a la ejecución de tareas, lo que trae como consecuencia directa un bajo rendimiento escolar. (Carvajal et al., 2006, p. 256)

La evidencia apunta que el estilo cognitivo de niños y niñas con TDAH difiere de los niños que no presentan este cuadro. Los estilos cognitivos se refieren a la forma específica en que las personas en forma particular perciben y procesan la información. Para el caso de niñez con TDAH es el estilo impulsivo el que predomina por sobre el reflexivo, lo que trae consigo ciertas consecuencias (Condemarín et al., 2008).

En cuanto a las dificultades que pueden enfrentar estudiantes con TDAH en el área del lenguaje en la enseñanza formal, se ha determinado que, respecto a la CL, alrededor de un $50 \%$ de niños y niñas con TDAH presenta problemas en la lectura y escritura (Miranda-Casas et al., 
2010). Es así como en investigaciones como la de Brock \& Knapp (1996) determinaron que el alumnado con TDAH mostró una menor competencia que el estudiantado del grupo control para identificar tópicos y las ideas centrales en una serie de textos presentados.

Por último, Miranda-Casas et al. (2010) realizan un estudio en donde se comparó niñez con TDAH y sin TDAH, con equilibrio en inteligencia, en reconocimiento de palabras, en comprensión literal e inferencial, así como también en la orden de fragmentos de textos. El hallazgo más notorio detectado por Miranda-Casas et al. (2010) es que ambos grupos no diferían significativamente en la comprensión literal e inferencial; no obstante, el estudiantado con TDAH mostraban un rendimiento significativamente menor a sus pares, en la tarea de colocar un orden a los fragmentos de textos, tarea que a juicio de los autores citados depende de la aplicación de habilidades de autorregulación, la organización de la información y el mantenimiento del esfuerzo sostenido, habilidades que se verían afectadas en niños, niñas y jóvenes con TDAH.

\section{Carácter del estudio}

El carácter de este estudio es del tipo comparativo-causal (Bisquerra, 2009), por tanto, se pretende determinar los efectos que tiene el TDAH en el desempeño en tareas de CL y PT en estudiantes de $4^{\circ}$ año de enseñanza básica. Se propone comparar a un grupo de estudiantes con TDAH y un grupo sin TDAH de un mismo nivel y medir el resultado por medio de un instrumento estandarizado igual para ambos grupos, aplicando esta prueba solo una vez y al mismo tiempo.

La presunción que existe detrás de esta comparación es que dados los antecedentes contextuales y los de la bibliografía revisada en el marco teórico de este estudio, se podría hipotetizar que el estudiantado con TDAH tendrá desempeños inferiores o más descendidos respecto a sus pares sin TDAH en tareas de CL y PT.

Lo anterior en el marco de un diseño de investigación, del tipo no experimental denominado ex post facto "después del hecho o de los hechos" (Bisquerra, 2009; Hernández, FernándezCollado y Baptista, 2010), en el cual el diagnóstico por TDAH no se puede manipular, ya que está dado; sin embargo, sí se pueden comparar los desempeños de dos grupos independientes y se tendería a atribuir desempeños más bajos en el estudiantado con TDAH, respecto de los que no lo presentan de acuerdo con el marco teórico ya presentado.

\section{Objetivos de la investigación}

\section{Objetivo general}

Comparar el desempeño de alumnos y alumnas con y sin la condición de TDAH en tareas de Comprensión de Lectura (CL) y Producción de Textos (PT) en estudiantes de $4^{\circ}$ año de enseñanza básica en Chile. 
doi: http://dx.doi.org/10.15359/ree.21-3.7

URL: http://www.una.ac.cr/educare

CORREO: educare@una.cr

\section{Objetivos específicos:}

- Medir el desempeño de alumnos y alumnas de $4^{\circ}$ año de enseñanza básica con la condición de TDAH en tareas de comprensión lectora $(\mathrm{CL})$.

- Medir el desempeño de alumnos y alumnas de $4^{\circ}$ año de enseñanza básica sin la condición de TDAH en tareas de comprensión lectora (CL).

- Medir el desempeño de alumnos y alumnas de $4^{\circ}$ año de enseñanza básica con la condición de TDAH en tareas producción de textos (PT).

- Medir el desempeño de alumnos y alumnas de $4^{\circ}$ año de enseñanza básica sin la condición de TDAH en tareas de producción de textos (PT).

\section{Hipótesis de investigación e hipótesis nulas}

$\mathrm{H}_{\mathrm{a}}$ : $\quad$ El estudiantado de $4^{\circ}$ año de enseñanza básica diagnosticados por TDAH obtendrá desempeños inferiores en tareas de $C L$ respecto a sus pares sin el diagnóstico.

$\mathrm{H}_{\mathrm{b}} \quad$ El estudiantado de $4^{\circ}$ año de enseñanza básica diagnosticado por TDAH obtendrá desempeños inferiores en tareas de PT respecto a sus pares sin el diagnóstico.

\section{Hipótesis nulas}

$\mathrm{H}_{\mathrm{o}} \quad$ El estudiantado de $4^{\circ}$ año de enseñanza básica diagnosticado por TDAH obtendrá desempeños iguales o similares en tareas de $C L$ respecto a sus pares.

$\mathrm{H}_{\mathrm{o}}$ : $\quad$ El estudiantado de $4^{\circ}$ año de enseñanza básica diagnosticado por TDAH obtendrá desempeños iguales o similares en tareas de PT respecto a sus pares.

\section{Diseño de investigación}

Para efectos de esta investigación, se tomarán los grupos intactos, ya que se evaluaron a 5 estudiantes de cada $4^{\circ}$ año básico que tienen el diagnóstico de TDAH ( 20 en total) y del otro estudiantado fueron seleccionados 5 de cada curso al azar, mediante el número de lista que ocupen (20 en total). El número de participantes que componen cada grupo corresponde a 20 estudiantes, con un total de 40 alumnos y alumnas $(\mathrm{N}=40)$.

La medición u observación fue aplicada solo una vez durante el segundo semestre del año 2014, específicamente y de acuerdo con el cronograma fue aplicada a todos los cursos en la primera semana de septiembre del 2014. La aplicación fue llevada a cabo en la clase de lenguaje y comunicación en cada curso.

\section{Muestra}

La muestra, tal como ya se había mencionado en el diseño, corresponde a 40 estudiantes de $4^{\circ}$ año básico de un colegio de dependencia particular subvencionada de la comuna de Macul, con diagnóstico de TDAH o sin este. 
El tipo de muestra que se ha elegido es del tipo no probabilístico o dirigida (Hernández et al, 2010), en la cual se tomará a un grupo de manera intacta y en el caso del estudiantado sin diagnóstico de TDAH la selección se hizo de manera aleatoria. Por tanto, en cada grupo habrá 5 estudiantes de cada curso con TDAH correspondientes a un total de 20 estudiantes, respecto a 5 estudiantes ${ }^{3}$ sin TDAH de cada curso (20 en total) que corresponderían al grupo de comparación o control.

\section{Análisis de datos}

\section{Plan de análisis}

Dado que la variable independiente $(\mathrm{VI})$ corresponde a la presencia o ausencia del diagnóstico por TDAH, referida a un nivel de medición nominal y a las variables dependientes que en esta investigación son el desempeño en tareas de $C L$ y PT, las que se encuentran en un nivel de medición intervalar, se empleará en primera instancia las herramientas de la estadística descriptiva como la media y la desviación estándar para cada uno de los grupos dentro de un mismo curso. Estos estadísticos descriptivos permiten visualizar de mejor forma la distribución y dispersión de los puntajes obtenidos luego de la aplicación del instrumento al estudiantado de los grupos control y experimental.

Cabe destacar que, en el caso de la $\mathrm{CL}$, el puntaje se encuentra definido por el instrumento y va desde 0 a 26 puntos y en el caso de la PT el puntaje se encuentra entre 0 y 39 puntos como máximo respectivamente.

Luego de presentar los estadísticos descriptivos, se hizo uso de las herramientas de la estadística inferencial y, para este caso en particular, la técnica empleada fue la de diferencia de medias, la que se calculó con base en la prueba estadística t de "Student" (Aron y Aron, 2001).

La prueba t de "Student" fue elaborada por Gosset (1908, citado por Aron y Aron, 2001), tiene como objetivo comparar las diferencias de medias (promedios entre dos grupos). En este caso se utilizará la prueba para muestras independientes, es decir, para sujetos que no están relacionados entre sí (Aron y Aron, 2001).

Esta prueba asume homocestacidad (varianzas homogéneas) y además el número de sujetos en cada muestra es igual (20 y 20). Adicionalmente, la variable independiente tiene un nivel de medición nominal y la variables dependiente (puntajes en CL y PT) son variables que están en el nivel de medición intervalar (Aron y Aron, 2001).

Esta prueba estadística asume, además, que la hipótesis nula $\left(\mathrm{H}_{\mathrm{o}}\right)$ señala que no hay diferencias estadísticamente significativas entre los desempeños del estudiantado con TDAH y quienes no presentan este cuadro en el desempeño de tareas de CL y PT.

${ }^{3}$ En el caso del grupo experimental se resguardó adicionalmente el criterio de que existiera equiparidad de género, ya que el grupo experimental estaba constituido mayoritariamente por varones (12 niños y 8 niñas). 
doi: http://dx.doi.org/10.15359/ree.21-3.7

URL: http://www.una.ac.cr/educare

CORREO: educare@una.cr

Para el caso del análisis de los resultados en el desempeño en las tareas de CL y PT se utilizó el programa estadístico SPSS, versión 15 y adicionalmente se calculó el estadístico "d" de Cohen para determinar el tamaño del efecto estadístico por medio del software GPower.

Se asumirá también que el grado de significación estadística que se considerará para retener la hipótesis de investigación y rechazar la hipótesis nula, tendrá un valor $p=0,05$. Por tanto, si en el análisis de diferencias de medias arroja un resultado igual o mayor que 0,05; no se podrá retener la hipótesis de investigación, ya que la probabilidad de que el resultado se dé por puro azar es mayor que 5 entre mil y en ese caso no se puede afirmar categóricamente que la hipótesis de investigación es cierta, sino que más bien se retendrá la hipótesis nula, que es lo que se supone a priori en una prueba de hipótesis.

En caso contrario, que el nivel de significancia arrojado por la prueba de diferencias medias sea mucho menor que 0,05, entonces se retendrá la hipótesis de investigación (Hi) y se rechazará la hipótesis nula (Ho).

\section{Resultados de los análisis}

Tal como se había señalado se realizarán un análisis comparativo en una primera instancia por medio de los estadísticos descriptivos, construidos luego de la aplicación de los instrumentos y la obtención de los puntajes en CL (0 -26) y en PT (0 - 39).

En la tabla 1 se muestran las medias y las desviaciones estándar para ambos grupos en $\mathrm{CL}$, de acuerdo con los resultados obtenidos en la prueba de CL-PT.

Tabla 1: Comparación de medias y desviaciones estándar grupo experimental y control en tareas de CL

\begin{tabular}{|c|c|c|c|c|c|}
\hline & & \multirow[b]{2}{*}{ TDAH } & \multicolumn{3}{|c|}{ Bootstrap ${ }^{a}$} \\
\hline & & & Estadísticos & Sesgo & Error estándar \\
\hline \multirow[t]{8}{*}{ Comprensión lectora } & Sin TDAH & $\mathrm{N}$ & 20 & & \\
\hline & & Media & 22,7250 &,- 0475 &, 5367 \\
\hline & & Desviación estándar & 2,48403 &,- 13174 & 47164 \\
\hline & & Media de error estándar & ,55545 & & \\
\hline & CONTDAH & $\mathrm{N}$ & 20 & & \\
\hline & & Media & 17,8250 & 0060 & 6317 \\
\hline & & Desviación estándar & 3,17173 &,- 05710 & ,40063 \\
\hline & & Media de error estándar & 70922 & & \\
\hline
\end{tabular}

a menos que se indique lo contrario, los resultados del bootstrap se basan en 100 muestrs de bootstrap. 
Según la Tabla 1, el estudiantado del grupo control (sin diagnóstico de TDAH) presenta un puntaje promedio o media de 22,73 puntos (de una máximo de 26), en tanto que el del grupo experimental alcanza un puntaje promedio de 17,83 (de un máximo de 26). De lo anterior se deduce que, efectivamente, el estudiantado sin el diagnóstico por TDAH obtiene puntuaciones mayores y desempeños más altos que sus pares con TDAH en tareas de comprensión lectora.

Adicionalmente, la desviación estándar (SD) en ambos grupos también difiere, más específicamente el estudiantado del grupo control tiene una SD de 2,48 mientras que el estudiantado del grupo experimental obtiene una SD de 3,17, lo que implica que los puntajes están más dispersos en el grupo experimental, es decir, existe mayor variabilidad en cuanto a puntajes entre sujetos del mismo grupo.

Respecto a los desempeños en PT, se presenta la Tabla 2 con los descriptores estadísticos.

Tabla 2: Tabla de medias y desviaciones estándar respecto al desempeño de tareas de PT de estudiantes con TDAH y sin TDAH

\begin{tabular}{|c|c|c|c|c|c|}
\hline \multirow[b]{2}{*}{ TDAH } & & & \multirow[b]{2}{*}{ Estadísticos } & \multicolumn{2}{|l|}{ Bootstrap $^{a}$} \\
\hline & & & & Sesgo & Error estándar \\
\hline \multirow[t]{8}{*}{ Producción de textos } & Sin TDAH & $\mathrm{N}$ & 20 & & \\
\hline & & Media & 29,6300 &,- 0071 & ,9430 \\
\hline & & Desviación estándar & 4,17588 &,- 26073 &, 75024 \\
\hline & & Media de error estándar & ,93376 & & \\
\hline & CON TDAH & $\mathrm{N}$ & 20 & & \\
\hline & & Media & 23,7000 &,- 0827 & 1,4329 \\
\hline & & Desviación estándar & 6,34201 &,- 26123 & ,60466 \\
\hline & & Media de error estándar & 1,41812 & & \\
\hline
\end{tabular}

a menos que se indique lo contrario, los resultados del bootstrap se basan en 100 muestrs de bootstrap.

Nuevamente se puede apreciar que los desempeños en términos de media son superiores en el caso del grupo control (sin diagnóstico de TDAH) con un puntaje promedio de 29,63 (de un máximo de 39 puntos). En tanto en el caso del estudiantado del grupo experimental la media es inferior, llega a 23,7 puntos (de 39 en total), se aleja en casi 6 puntos en promedio, respecto al grupo control, y alcanza menores puntajes en promedio en tareas de PT.

Respecto a la desviación estándar en ambos grupos, en el grupo experimental nuevamente existe unSD mayor $(6,34)$ queenel grupo control $(S D=4,18)$, lo queimplica que en grupo diagnosticado por TDAH existe mayor dispersión y variabilidad en los puntajes obtenidos en las tareas de PT. 
doi: http://dx.doi.org/10.15359/ree.21-3.7

URL: http://www.una.ac.cr/educare

CORREO: educare@una.cr

Para los objetivos de la investigación, hasta ahora se puede visualizar que, efectivamente, el estudiantado del grupo experimental (conTDAH) obtiene desempeños más descendidos que el alumnado sin TDAH. Lo anterior reafirma la hipótesis de investigación de este estudio, la cual hace alusión a la comparación de los desempeños de ambos grupos, con resultados inferiores en ambas tareas del grupo experimental.

Sin embargo, para los fines de investigación se debe establecer si las diferencias de medias son estadísticamente significativas para rechazar la hipótesis nula y aceptar la hipótesis de investigación, la que señala, según la bibliografía revisada que el estudiantado con TDAH tendrá rendimientos más descendidos que sus pares sin este diagnóstico. Para ello se empleará a continuación la prueba t de "Student" para muestras independientes, para evidenciar si estas diferencias de medias responden a la hipótesis de investigación.

A continuación, se presenta la Tabla 3 con los resultados de la prueba de t de Student para muestras independientes, respecto al desempeño en tareas de $\mathrm{CL}$.

Tabla 3: Prueba de t de Student para comparar medias de muestras independientes respecto a desempeño en tareas de $\mathrm{CL}$

\begin{tabular}{cccccc}
\hline & $\mathrm{F}$ & Diferencias de medias & $\mathrm{t}$ & $\mathrm{gl}$ & Sig (Bilateral) \\
\hline Comprensión lectora & 1,505 & 4,900 & 5,439 & 38 & 0,000 \\
\hline
\end{tabular}

Tal como se aseveraba con anterioridad, la prueba t de Student informa que existe significancia estadística, que se puede visualizar en la columna de significancia bilateral, la cual es menor a $\mathrm{p}=0,05$ (sig.bilateral $=0,00$ ) respecto a la comparación de medias entre ambos grupos. Es decir, se puede aseverar que los resultados obtenidos del desempeño del estudiantado con TDAH, respecto al alumnado sin TDAH, confirman la hipótesis de investigación, y señalan que efectivamente el cuadro de TDAH incide en el desempeño de tareas de $C L$, que es lo que se quería probar mediante la prueba estadística.

Por tanto, se puede constatar que existen diferencias estadísticamente significativas respecto a los puntajes obtenidos por el grupo control con $\mu=22,73$ y $S D=2,48$ y el grupo experimental con $\mu=17,83$ y $S D=3,17$ y $(t=5,43 ; g l=38)$. Asumiendo que el nivel de significancia obtenido fue de 0,05 , la Tabla 3 arroja una significancia bilateral de 0,0. Por tanto, se rechaza la hipótesis nula y se acepta la hipótesis de investigación. Es decir, el diagnóstico con TDAH incide en las tareas de CL.

Tabla 4: Prueba de t de Student para comparar medias de muestras independientes respecto a desempeño en tareas de PT

\begin{tabular}{cccccc}
\hline & $\mathrm{F}$ & Diferencias de medias & $\mathrm{t}$ & $\mathrm{gl}$ & Sig (Bilateral) \\
\hline Producción de textos & 8,286 & 5,93 & 3,492 & 38 & 0,001 \\
\hline
\end{tabular}


En la Tabla 4 se puede apreciar que existe significancia estadística, en relación con el desempeño del estudiantado del grupo control respecto al experimental. Se obtiene para el grupo control una $\mu=29,63$ y una $S D=4,17$ en tanto para el grupo experimental, $\mu=23,7$ y $S D=$ $6,34(t=3,49 ; g l=38)$ con un valor $p=0,05$. La Tabla 4 de significancia bilateral arroja un resultado de 0,01 . Lo que permite rechazar la hipótesis nula y aceptar la hipótesis de investigación, es decir, el diagnóstico por TDAH afecta el desempeño de tareas de PT.

Por último, dentro de este apartado, se busca determinar la potencia estadística del estudio, dado que el número de estudiantes es reducido, en virtud de la cantidad de estudiantes que reciben diagnósticos y certificados son escasos respecto al resto de la población escolar, para este contexto particular.

Se definirá potencia estadística como la probabilidad de que un estudio tenga un resultado significativo, es decir, para determinar si la hipótesis de investigación es verdadera y de cumplir con los objetivos planteados en el estudio (Aron y Aron, 2001).

Para ello se calculará el tamaño del efecto por medio de la "d" de Cohen, la cual tiene que tener un valor igual o superior a 0,8 para que el tamaño del efecto de esta investigación sea grande o significativo.

Para el caso de la CL, empleando el software estadístico GPower, el tamaño del efecto arroja un resultado de un tamaño del efecto $\mathrm{d}=1,5 \mathrm{y}$ en el caso de las tareas de PT el resultado que arroja un tamaño del efecto $d=0,94$. Ambos resultados señalan que la potencia del estudio es grande y que, por tanto, se cumplen los objetivos y se ratifican las hipótesis de investigación con un nivel de significancia de $\mathrm{p}=0,05$; no obstante la reducida muestra que se consideró en este estudio con un total de 40 estudiantes, agrupados en 20 alumnos y alumnas con TDAH y $20 \sin$ TDAH.

\section{Discusión}

El presente estudio perseguía comparar los desempeños del estudiantado con la condición de déficit atencional y sin esta de $4^{\circ}$ año de educación en tareas de $C L$ y PT. De acuerdo con los resultados arrojados por el instrumento de evaluación, se confirmó la hipótesis de investigación, ya que el alumnado diagnosticado por TDAH obtuvo rendimientos inferiores en tareas de $\mathrm{CL}$ y PT que sus pares sin este cuadro.

Luego de revisar de manera más intensiva la bibliografía existente, en la cual se aseveraba que los rendimientos de los niños y las niñas con TDAH tenían un menor desempeño en tareas asociadas al lenguaje en general, sin centrarse específicamente en tareas de comprensión lectora o de producción de textos como tal. En este sentido, los resultados reportados por los estadísticos descriptivos y la prueba t de Student confirman la hipótesis de investigación en este grupo experimental respecto al grupo de control, evidenciándose diferencias estadísticamente 
doi: http://dx.doi.org/10.15359/ree.21-3.7

URL: http://www.una.ac.cr/educare

CORREO: educare@una.cr

significativas en los desempeños del estudiantado con TDAH respecto a los que no están diagnosticados por este cuadro.

Los resultados de esta investigación confirman lo expresado de manera genérica en los estudios internacionales y nacionales en cuanto a la incidencia de este cuadro en el desarrollo del lenguaje, pero profundiza algunos aspectos fundamentales en la educación escolar, al detallar las que corresponden a la comprensión de lectura y la producción de textos escritos por parte del alumnado. Tareas de alta complejidad y que requieren seguir siendo estudiadas más en detalle y ofrecer, a la vez, herramientas específicas a los grupos pedagogos para poder intervenir en estas áreas, con el fin de brindar un apoyo más focalizado en las necesidades específicas de los niños y las niñas que han recibido diagnóstico por TDAH.

Como una forma de seguir profundizando por medio de futuras investigaciones en esta línea de estudios, se proponen preguntas que pueden ser materia de futuros hallazgos y debates y que, además, podrían aportar mayores antecedentes para mejorar el apoyo pedagógico al estudiantado con dificultades en el área atencional y del lenguaje. Interrogantes como, por ejemplo: ¿Existen diferencias entre el rendimiento de los subtipos de déficit atencional, es decir, entre aquellos que poseen más hiperactividad que inatención o los que tienen mayores niveles de atención que de hiperactividad?

Respecto al género: ¿Existen diferencias estadísticamente significativas en el desempeño en tareas de $\mathrm{CL}$ y PT entre niños y niñas que han recibido diagnóstico por este cuadro?

En cuanto a variables como nivel socioeconómico (NSE): ¿Existen diferencias estadísticamente significativas entre niñez con TDAH en escuelas particulares pagadas y municipalizadas (estatales)? ¿Se pueden atribuir estas diferencias solo al TDAH o se ven afectadas por el NSE del estudiantado?

\section{Referencias}

Aboitiz, F., López-Calderón, J., López, V. Galaburda, A. M. (2006). Neurobiología del déficit atencional: Endofenotipos y dinámica de los sistemas dopaminérgicos En I. López, V. Boehme, J. Förster (Eds.), Síndrome de déficit atencional (pp. 27-44). Santiago: Editorial Universitaria.

Agencia de Calidad, Gobierno de Chile. (2013). SIMCE 2012: Síntesis de Resultados $4^{\circ}$ básica. Recuperado de http://www.agenciaeducacion.cl/wp-content/uploads/2013/06/ SR 4\%C2\%BAbasico 2012 web.pdf

Agencia de Calidad, Gobierno de Chile. (2016). SIMCE 2013: Síntesis de Resultados $4^{\circ}$ Educación básica. Recuperado de https://s3.amazonaws.com/archivos.agenciaeducacion.cl/ resultados-simce-2013/SR 4basico 2013.pdf 
Aron, A. y Aron, E. N. (2001). Estadística para psicología. Buenos Aires: Pearson Education.

Bisquerra, R. (Coord.). (2009). Metodología de la investigación educativa (2a ed.). Madrid: La Muralla.

Brock, S. y Knapp, P. K. (1996). Reading comprehension abilities of children with Attention Deficyt/Hiperactivity. Journal of Attention Disorders, 1(3), 173-185. doi: https://doi. org/10.1177/108705479600100305

Cain, K., Oakhill, J. y Bryant, P. (2004). Children's reading comprehension ability: Concurrent prediction by working memory, verbal ability, and component skills. Journal of Educational Psicology, 96(1), 31-42. doi: https://doi.org/10.1037/0022-0663.96.1.31

Carvajal, M., Cabezas, M, y Valenzuela, G. (2006). Intervención a nivel escolar de niños y adolescentes con síndrome de déficit atencional. En I. López, V. Boehme, J. Förster (Eds.), Síndrome de déficit atencional (pp. 251-274). Santiago: Editorial Universitaria.

Condemarín, M., Gorostegui, M. E. y Milicic, N. (2008). Déficit atencional. Estrategias para el diagnóstico y la intervención psicoeducativa ( $5^{\mathrm{a}}$ ed.). Santiago: Ariel.

Cuetos, F. (2009). Psicología de la escritura ( $8^{\mathrm{a}}$ ed.). Madrid: Wolters Kluwer.

Edel, R. (2003). El rendimiento académico:Concepto, investigación y desarrollo. RevistaElectrónica Iberoamericana sobre Calidad, Eficacia y Cambio en Educación, 1(2), 1-16. Recuperado de http://www.redalyc.org/articulo.oa?id=55110208

Elías, J., Crespo, N.y Góngora, B. (2012). El desempeño sintáctico de niños con trastorno de déficit atencional e hiperactividad: Perspectiva comparativa y ontogenética. Revista de Lingüística Teórica y Aplicada, 50(1), 95-117. doi: https://doi.org/10.4067/S0718-48832012000100005

Faraone, S. V., Sergeant, J., Gillberg, C. y Biederman, J. (2003). The worldwide prevalence of ADHD: is it an American condition? World Psychiatry, 2(2). 104-113. Recuperado de https:// www.ncbi.nlm.nih.gov/pmc/articles/PMC1525089/

García, M. V., Alvarado, J. M. y Jiménez, A. (2000). La predicción del rendimiento académico: Regresión lineal versus regresión logística. Psicothema, 12(2), 248-252. Recuperado de http://www.psicothema.com/pdf/558.pdf

Hernández, R., Fernández-Collado, C. y Baptista, P. (2010). Metodología de la investigación (5ª ed.). México: McGraw-Hill.

López, I. (2006). Introducción. En I I. López, V. Boehme, J. Förster (Eds.), Síndrome de déficit atencional ( $3^{\mathrm{a}}$ ed., pp. 13-22). Santiago: Editorial Universitaria. 
doi: http://dx.doi.org/10.15359/ree.21-3.7

URL: http://www.una.ac.cr/educare

CORREO: educare@una.cr

Medina, A.y Gajardo, A. M. (2010). Pruebas de comprensión lectora y producción de textos (CL-PT), Kínder a $4^{\circ}$ Básico. Marco conceptual y manual de aplicación y corrección. Santiago: Ediciones UC.

Miranda-Casas, A., Fernández-Andrés, P., Robledo, P. y García-Castellar, R. (2010). Comprensión de textos de estudiantes con trastorno por déficit de atención/hiperactividad: ¿Qué papel desempeñan las funciones ejecutivas? Revista Neurología, 50(3), S135-S142. Recuperado de http://www.revneurol.com/sec/resumen.php?id=2009768\#

Rubio, M. (2011). Concepciones sobre la producción escrita en académicos que forman profesores básicos. Literatura y Lingüística, 23, 123-140. doi: https://doi.org/10.4067/ $\underline{\text { S0716-58112011000100008 }}$

Universidad de Chile, Centro Micro Datos (2011). Estudio sobre el comportamiento lector a nivel nacional. Santiago, Chile: Autor.

Vallés, A. (2005). Comprensión lectora y procesos psicológicos. Liberabit, 11(11), 49-61 Recuperado de http://pepsic.bvsalud.org/pdf/liberabit/v11n11/v11n11a07.pdf 